\title{
Ist der Diversifikationsvorteil bei Teams nachhaltiger als am Finanzmarkt?
}

\section{Oder: Tough Times Don't Last, Tough Teams Do!}

DOI 10.1007/s11576-010-0212-8

\section{Der Autor}

Prof. Dr. Hans Ulrich Buhl ( $\varangle)$

FIM Kernkompetenzzentrum Finanz-

\& Informationsmanagement

Universität Augsburg

Universitätsstraße 12

86159 Augsburg

Deutschland

hans-ulrich.buhl@

wiwi.uni-augsburg.de

Online publiziert: 2010-02-25

This article is also available in English via http://www.springerlink.com and http://www.bise-journal.org: Buhl HU (2010) Is the Diversification Benefit for Teams More Sustainable Than at the Financial Market? Or: Tough Times Don't Last, Tough Teams Do! Bus Inf Syst Eng. doi: 10.1007/s12599010-0091-y.

(c) Gabler Verlag 2010
Die seit Juli 2007 anhaltende Finanzmarktkrise war in Gesellschaft, Politik, Wissenschaft und Praxis das dominierende Thema der letzten beiden Jahre. So befassten sich unzählige Beiträge und Diskussionen unter anderem in Zeitungen, Rundfunk und Fernsehen sowie in wissenschaftlichen Journals mit Hintergründen, Ursachen, Lösungsansätzen, möglichen Zukunftsszenarien und vielen weiteren Aspekten der Krise. Dies erscheint auch angebracht, hat doch die Wirtschaftskrise laut der Internationalen Arbeitsorganisation (ILO) weltweit mindestens 20 Mio. Arbeitsplätze vernichtet. „Bei einem vorzeitigen Ende staatlicher Konjunkturprogramme könnten bis zu 43 Mio. Arbeitsplätze gefährdet sein“. (o. V. 2009b) Darüber hinaus bezifferte eine Studie von Commerzbank Research die weltweiten Verluste durch die Krise auf weit über zehn Billionen US-Dollar (o. V. 2009c). Um sich die Dimensionen dieses Betrags einmal ansatzweise vorstellen zu können: Wenn man an jedem von 200 Arbeitstagen im Jahr einen 1000 US-Dollar-Schein verbrennt, benötigt man 50 Mio. Jahre, um diesen Betrag zu vernichten. Zum Vergleich: Vor ca. 50 Mio. Jahren hat der Zusammenstoß der afrikanischen und der europäischen Kontinentalplatte die Alpen mit ihren Viertausendern wie Matterhorn, Monte Rosa und Jungfrau entstehen lassen. Als bislang älteste Überreste des modernen Menschen gelten Schädelknochen des Homo sapiens idaltu aus dem heutigen Äthiopien, welche auf ein Alter von 160.000 Jahre datiert werden (o. V. 2003). Folglich wird für die Vernichtung eines solchen Betrags in obigem Beispiel ein Zeitraum benötigt, der dem über 300-fachen der heute bekannten Menschheitsgeschichte entspricht. Und ja, auch wir werden das aktuelle Editorial diesem Thema widmen. Denn trotz aller Beiträge und Diskussionen bleiben wirklich nachhaltige Lösungsansätze zur Vermeidung zukünftiger Krisen nach wie vor eher Mangelware. Und noch schlimmer: Nicht einmal erkennbare Lehren scheinen insbesondere die Führungskräfte im Finanzsektor aus der aktuellen Krise gezogen zu haben. So wurde beispielsweise im Juli 2009 bekannt, dass Goldman Sachs und Barclays Capital eine neue Variante von CDOs (Collateralized Debt Obligations - eine Variante von mehrfach strukturierten Wertpapieren, deren Werte im Zuge der Finanzkrise abgestürzt sind) zur Erleichterung riskanter Anlagen entwickeln, welche die Kapitalkosten um bis zu 50 \% senken. Der Unterschied zu den bisherigen CDOs sei, dass es sich um die Verbriefung von Aktiva handelt, welche die Banken bereits in ihren Büchern halten, und nicht um Neugeschäft. „Elegante Verbriefung“ bezeichnet Barclays diese neue Methodik (Jenkins 2009). Klingt erschreckend vertraut, oder? Und das in einer Zeit, in der sich viele Banken trotz staatlicher Hilfen in Milliardenhöhe noch nicht richtig erholt haben. Spätestens an dieser Stelle darf man dann doch einmal die Frage stellen, welchen Bezug zur Realität so mancher Bankenmanager denn eigentlich noch hat. Eine ebenso erschreckende wie denkwürdige Demonstration von offensichtlichem Realitätsverlust gab Lloyd Blankfein, seines Zeichens CEO von Goldman Sachs, in einem Interview mit der Sunday Times im November 2009. Die aktuell wieder auf eine kaum vermittelbare Höhe steigenden Gehälter und Boni seines Hauses rechtfertigte er mit der Begründung, dass Banken Firmen, die wachsen wollen, bei der Aufnahme von Kapital helfen würden. Wachsende Firmen würden wiederum Wohlstand schaffen, sodass die Banken einem sozialen Zweck dienen und damit "Gottes Werk“ verrichten würden (o. V. 2009e). Goldman Sachs war sowohl für die Entstehung der Krise mitverantwortlich und hat mit ethisch fragwürdigen Geschäftspraktiken sogar in Milliardenhöhe an ihr verdient. Angesichts dieser Entwicklung darf man sich eigentlich nicht wundern, dass insbesondere die öffentliche Diskussion über Verantwortlichkeiten sowie über moralische und ethische Aspekte der 
Krise zunehmend emotionaler geführt wird. Aber worin liegt die Ursache? Natürlich könnte man sich hier nun sehr leicht der stellenweise wahrnehmbaren Verteufelung eines ganzen Berufstandes oder sogar einzelner Personen anschließen. Dies wäre aber weder konstruktiv noch zielführend, geschweige denn angebracht. Wo sind also Ansatzpunkte zu finden?

Eine mögliche Antwort könnte ein Blick auf die Zusammensetzung vieler Führungsgremien geben. Bei genauerer Betrachtung fällt auf, dass die Vorstände und Aufsichtsräte vieler großer Unternehmen sehr homogen bezüglich des Geschlechts und des fachlichen Backgrounds der Mitglieder besetzt sind. So bestand beispielsweise das elfköpfige Board of Directors der damals viertgrößten amerikanischen Investmentbank Lehman Brothers, die durch ihre Insolvenz am 15. September 2008 im Zuge der Finanzmarktkrise traurige Berühmtheit erlangte, ausschließlich aus Männern (Thorborg 2009). Dies ist aber kein Einzelfall. Lediglich 1,3 \% aller Vorstandsmitglieder der deutschen Top100-Unternehmen sind derzeit weiblich. In den Aufsichtsräten liegt der Frauenanteil mit 9,6 \% zwar etwas höher, jedoch sind davon ca. $60 \%$ Arbeitnehmervertreterinnen. Die deutsche Wirtschaft ist also nach wie vor „oben ohne“ (Stauber-Klein 2006). Auch in Österreich und der Schweiz sieht das Bild nicht viel besser aus (o. V. 2009a, S. 30). In den USA sind immerhin $16 \%$ der Vorstände in den 500 größten US-Unternehmen weiblich (Stauber-Klein 2006). Dabei deuten zahlreiche Studien darauf hin, dass gerade ein erhöhter Testosteronspiegel zu einer höheren Risikobereitschaft führt (o. V. 2009d). Und welche immensen, ja teilweise existenzbedrohenden Risiken so manche von Männern dominierten Führungsgremien der Banken vor Ausbruch der Finanzmarktkrise eingegangen sind, braucht an dieser Stelle wohl nicht mehr diskutiert werden.

Darüber hinaus besitzen beispielsweise ca. 88 \% der Mitglieder von Führungsgremien schweizerischer Unternehmen im Finanzsektor einen rein betriebswirtschaftlichen Hintergrund. Nur $10 \%$ können eine naturwissenschaftliche und $2 \%$ eine geisteswissenschaftliche Ausbildung vorweisen (Rost u. Osterloh 2008). An dieser Stelle könnte man das Argument anführen, dass insbesondere im Finanzsektor ja auch überwiegend betriebswirtschaftliche Fähigkeiten von Relevanz sind. Dies greift jedoch zu kurz. Zahlreiche psychologische Studien unterstützen die These, dass Experten in ihrem jeweiligen Fachgebiet öfters Opfer der berühmten Betriebsblindheit werden. Genauer gesagt überschätzen Experten oft die Genauigkeit der ihnen vorliegenden Informationen und ziehen daraus falsche Schlüsse hinsichtlich der Schätzung von Wahrscheinlichkeiten, mit der gewisse Ereignisse eintreten können (Rost u. Osterloh 2008, S. 8 f). Ein ähnliches Bild ergab sich ja auch während der Finanzmarktkrise. Ganz offensichtlich haben nahezu alle Marktteilnehmer ungeachtet der problematischen Anreizstrukturen die tatsächlichen Risiken der Wertpapiere und Verbriefungsstrukturen fehlerhaft bewertet. Alleine über eine erhöhte Risikobereitschaft ist es wohl nicht zu erklären, dass so viele Marktteilnehmer in teilweise beträchtlichem und existenzbedrohendem Umfang in die strukturierten Finanztitel investiert haben. Sogar Joseph Stiglitz, Nobelpreisträger und Professor von der Columbia-Universität in New York, meinte im August 2008: „Die Banken behaupten, sie könnten Risiken managen. Aber dazu sind sie nicht in der Lage. Sie sind nicht einmal in der Lage, ihre Risiken richtig zu beziffern. Es ist phänomenal, wie sehr die Banken danebengelegen haben (Schäfer 2008)“.

Aber was ist nun die Schlussfolgerung und, für die Zukunft noch viel wichtiger, die Lösung? Hätte die Finanzmarktkrise verhindert werden können, wären weniger homogen ausgebildete Männer und mehr interdisziplinär ausgebildete Frauen an der Wall Street beschäftigt gewesen? Sind Frauen pauschal die besseren Führungskräfte? Sollten zukünftig nur noch interdisziplinär ausgebildete Mitarbeiter für Führungspositionen in Betracht gezogen werden? Zugespitzt formuliert: Sollte die Leitung von Banken und anderer Großunternehmen also zukünftig ausschließlich in die Hände von Wirtschaftsinformatikerinnen und Wirtschaftsingenieurinnen gelegt werden? Und wie muss man sich dies dann vorstellen? Wären, mal äußerst überspitzt und provokativ gesprochen, beispielsweise die Wall Street und die Londoner City dann wundervolle Orte mit vielen kleinen Cafés, in denen bei Latte Macchiato und kalorienarmen Himbeertörtchen bevorzugt über Skype und ICQ alle Arten von Beziehungen gepflegt werden? Wohl kaum.

Beschäftigt man sich etwas näher mit dieser Thematik, bleibt eigentlich nur eine ernsthafte und nachhaltige Schlussfolgerung und Lösung übrig. Das tatsächliche Problem scheinen nämlich weder die Männer noch die Experten an sich darzustellen, sondern die Homogenität der Führungsgremien. Eine erhöhte Risikobereitschaft insbesondere im unternehmerischen Umfeld scheint keineswegs negativ, ganz im Gegenteil. Wie sagt man doch: „Keine Chance ohne Risiko“. Fehlt jedoch in manchen Si- 
tuationen die kritische und mäßigende Gegenstimme, sozusagen der advocatus diaboli (welcher natürlich auch ein interdisziplinär ausgebildeter Mann sein kann), dann besteht die Gefahr, dass übermäßig hohe Risiken teils unreflektiert und ohne vorausgehende, kontroverse Diskussion - ja vielleicht sogar in Unkenntnis - eingegangen werden. Genau dies haben wir am Beispiel der Entstehung der Finanzmarktkrise und ihrem ebenfalls durch homogenes Verhalten so raschem Platzen der Marktblasen gesehen. Und selbstverständlich werden auch im Finanzsektor immer Führungskräfte mit betriebswirtschaftlichem Expertenwissen erforderlich sein. Jedoch kann es für ein Unternehmen äußerst gesund sein, wenn insbesondere in Phasen der beginnenden Übertreibung einige Nicht-Experten vielleicht sogar etwas laienhaft und naiv das entstehende Herdenverhalten kritisch hinterfragen und mit gesunden Menschenverstand versuchen, die eventuell vorherrschende Betriebsblindheit der Experten aufzulösen. In der Land- und Forstwirtschaft ist schon lange bekannt, dass insbesondere Monokulturen anfällig für Krankheiten und für die Ausbreitung spezialisierter Schädlinge sind. Ein durchaus nachhaltiger Lösungsansatz wäre also der Wandel von Monokulturen hin zu Mischkulturen oder, um der zugegebenermaßen durchaus provokativen Metapher wieder den Rücken zu kehren, die Heterogenisierung der Führungs- und Kontrollgremien. Selbst Rolf Breuer, ehemaliger Vorstandsvorsitzender und Sprecher des Aufsichtsrats der Deutschen Bank, formulierte das oftmals in Aufsichtsräten anzutreffende „Lemming-Verhalten“ als einen Grund, warum die Finanzmarktkrise viele Banken so kalt erwischt hat (Thorborg 2009).

Und tatsächlich zeigen beispielsweise Studien des Beratungsunternehmens McKinsey und der amerikanischen Lobbyorganisation Catalyst, dass die Unternehmen mit den meisten Frauen im Vorstand im Vergleich zu solchen ohne Frauen weitaus erfolgreicher agierten und eine bis zu 53 \% höhere Eigenkapitalrendite erzielten (Thorborg 2009). Zudem weisen auch die Cranfield University, die renommierte London School of Economics sowie die Weltbank auf diverse Vorteile der sich ergänzenden Führungsstile von Männern und Frauen hin (o. V. 2009a, S. 30). Darüber hinaus bestärken einige wissenschaftliche Arbeiten, dass Firmen eine größere Heterogenität ihrer Führungsgremien nicht nur über einen höheren Frauenanteil herstellen sollten, sondern insbesondere auch über die Einbindung mehrerer Mitglieder mit einem nicht rein betriebswirtschaftlichen Hintergrund. Denn abgesehen von einer grundsätzlich unterschiedlichen Risikobereitschaft scheinen nicht nur Frauen im Vergleich zu Männern in Situationen der Unsicherheit bessere Einschätzungen geben zu können, sondern auch Nicht-Experten im Vergleich zu Experten (Rost u. Osterloh 2008).

Und vielleicht sind diese Zusammenhänge auch gar nicht auf Unternehmen beschränkt. Zwar sind uns entsprechende Studien aus dem Hochschulbereich nicht bekannt, sollen aber hiermit angeregt werden. Vielleicht käme dabei ja beispielsweise heraus, dass der oft deutlich überdurchschnittliche Erfolg von WirtschaftsinformatikLehrstühlen in ihren Fakultäten bezüglich vieler Leistungsindikatoren weniger in der Qualität der Professor(inn)en begründet liegt als in der größeren Heterogenität ihrer Lehrstuhlteams. Und vielleicht zeigen solche Untersuchungen in Wissenschaft und Praxis, dass dieser Diversifikationsvorteil von Teams deutlich nachhaltiger und krisenresistenter ist als der Diversifikationsvorteil der Portfoliotheorie, für den Markowitz 1990 den Nobelpreis bekam. Dieser Diversifikationsvorteil löste sich dadurch, dass die Korrelationen vieler Assets auf nahezu +1 sprangen, in der Krise in vielen Portfolios weitgehend auf. Ein solcher Diversifikationseffekt, der in der Krise verschwindet, ist in etwa so viel wert wie eine Versicherung, die im Schadensfall nicht zahlt, nämlich deutlich weniger als Null. Wir wagen daher die These, dass team diversity asset diversity schlägt. Am besten ist vermutlich beides zusammen.

Was ist also aus heutiger Sicht konkret zu tun?

- Aus gesellschaftlicher Sicht wäre es wünschenswert, einen Bewusstseinswandel in den Köpfen aller Beteiligten und auf allen gesellschaftlichen Ebenen zu schaffen, um die Chancengleichheit von Männern und Frauen, disziplinär und interdisziplinär ausgebildeter Führungskräfte gleichermaßen zu fördern. Dabei gilt es einerseits, traditionell-rollenspezifische Einstellungen und Verhaltensweisen zu hinterfragen, um das beruflich-fachliche Potenzial insbesondere von Frauen zu stärken. Fraglich bleibt in Bezug auf den Anteil an Frauen in Führungsgremien dennoch, inwieweit dies in der Zukunft gelingen kann, solange die meisten Topmanager ihre Frau samt Kindern zu Hause lassen und damit die eigene familiäre Prägung der Männer in den Chefetagen eine zentrale Rolle spielt. Eine noch viel wichtigere Herausforderung ist andererseits, eine gesellschaftliche Akzeptanz für eine größere Heterogenität 
in Teams zu schaffen. Denn nur auf diese Weise kann die Basis gelegt werden, den Korpsgeist von überwiegend männlichen, fachlich homogenen und meist gleichgeschalteten Top-Management-Gruppen zu durchbrechen und eine für die Gesellschaft wünschenswerte Vielfalt an Denkmustern zu fördern.

- Und was kann die Politik an dieser Stelle leisten? Die Politik ist vor allem aufgefordert, die Rahmenbedingungen für eine noch stärkere Förderung von Frauen in Führungspositionen zu schaffen. Maßnahmen wie beispielsweise der Ausbau der Kinderbetreuung oder die bessere steuerliche Absetzbarkeit von Kinderbetreuungskosten sind nur einige Beispiele dafür, wie Frauen in ihrer Karriere stärker unterstützt werden können. Ob das niederländische Modell mit einer gesetzlich vorgeschriebenen Frauenquote von 30 \% in Führungs- und Kontrollgremien dabei der richtige Weg ist, sei mal dahingestellt (auch wenn diese im Hinblick auf die Frauenförderung sehr erfolgreich sind; Haas u. Kur 2009). Aber wir dürfen auch in Deutschland hoffen: im Koalitionsvertrag der neuen Bundesregierung heißt es, dass der Anteil von Frauen in Führungspositionen in der Wirtschaft und im öffentlichen Dienst in der jetzigen Legislaturperiode maßgeblich erhöht werden soll. Ob dies nur ein Lippenbekenntnis darstellt, wird sich in naher Zukunft herausstellen. Jedenfalls betont Ursula von der Leyen, Bundesministerin für Arbeit und Soziales, in einem ihrer ersten Interviews: Die bisherige Arbeitswelt war „mittelalt und männlich“, die neue Arbeitswelt wird „älter, weiblicher, bunter, innovativer sein“ (Öchsner 2009). Darüber hinaus ist es als zentrale Herausforderung im Rahmen der Bildungspolitik zu sehen, noch deutlich stärker die interdisziplinäre und Soft-Skill-Ausbildung von zukünftigen Führungskräften auszubauen. Dabei sollten einerseits in der universitären Lehre entsprechende Schwerpunkte wie beispielsweise Soft-Skill-Seminare oder interdisziplinäre Curricula verstärkt gesetzt werden. Andererseits ist auch über einen Ausbau extracurricularer Angebote - ein solches stellt beispielsweise bereits heute die Bayerische EliteAkademie dar - nachzudenken.

- Einen ganz entscheidenden Beitrag zur Förderung der Heterogenität in Führungsgremien können Unternehmen selbst leisten. Chancengleichheit sowohl bezüglich des Geschlechts als auch hinsichtlich der fachlichen Ausbildung sollte dabei als oberste Maxime gelten. So sollten beispielsweise unterschiedliche Gehälter zwischen Männern und Frauen oder auch das Phänomen „gläserner Decken“, die dazu führen, dass Männer im Topmanagement lieber unter ihres gleichen bleiben, in erfolgreich und nachhaltig geführten Unternehmen keine Chance haben. Darüber hinaus müssen Unternehmen eine Kultur schaffen, in der kritische Fragen und eine konstruktive Diskussionsbereitschaft auf allen Ebenen und Ebenen übergreifend jederzeit zugelassen - ja sogar erwünscht sind. Dabei sind auch Nicht-Experten in allzu disziplinären Diskussionen als vollwertige Diskussionsteilnehmer mit einzubinden. Wird eine unter Umständen kritische Meinung eines Diskussionsteilnehmers alleine aufgrund seines eventuell weniger stark ausgeprägtem Expertenwissens in einem bestimmten Bereich nicht ernst genommen, schadet sich das Unternehmen letztendlich nur selbst. Auch implizite Karrierekriterien sollten von Unternehmen expliziert werden. So kommt eine amerikanische Studie zu dem ernüchternden Ergebnis, dass oftmals der Erfolg einer Person letztendlich stärker von deren Durchsetzungsfähigkeit, Beharrlichkeit, Detailversessenheit und Effizienz als von deren Teamorientierung abhängig ist. Stärken in der Menschenführung haben fast nichts mit der Wahrscheinlichkeit für einen Aufstieg in die Führungsetage zu tun (Thorborg 2009). Als Konsequenz kann dies mit deutlich höherer Wahrscheinlichkeit zu gleichgerichteten Führungsgremien führen, die öfters einer Betriebsblindheit zum Opfer fallen. Unternehmen sind hierbei folglich aufgefordert, eine Unternehmenskultur zu schaffen, die eben nicht egozentrische und eindimensionale, sondern flexible, teamorientierte, sensible, empathische, gutausgebildete und vor allem für andersartige Denkweisen und Meinungen offene Führungskräfte - Männer wie Frauen - fördert.

- Eine wichtige Aufgabe hat aber auch die Wissenschaft in dieser Thematik, da ihr die Ausbildung der Nachwuchsführungskräfte von morgen zukommt. Was kann diese folglich hierzu beitragen? Die Bewusstseinsbildung der Gesellschaft über wissenschaftliche Studien bezüglich der Vorteilhaftigkeit heterogener Führungsgremien ist natürlich nur ein Thema. Dies scheint leider aber auch in den Hochschulen selbst nur graue Theorie geblieben zu sein, wenn man den Anteil an Akademikerinnen betrachtet, die eine wissenschaftliche Karriere anstreben. Hier gilt es ebenso wie in der Praxis, attraktive Modelle für Frauen zu schaffen und damit die leider viel zu geringe 
Anzahl an Professorinnen zu erhöhen. Auf diese Weise könnte auch der in manchem Professorium erhöhte durchschnittliche Testosteronspiegel deutlich zum Vorteil einer Universität gesenkt werden. Eine weitere ganz wesentliche Herausforderung der Wissenschaft besteht insbesondere in der interdisziplinären Ausbildung von Studenten und Doktoranden, um genau solche Persönlichkeiten auszubilden, die den Blick über den Tellerrand wagen. So muss einerseits die Ausbildung in von Hause aus interdisziplinären Fachrichtungen wie der Wirtschaftsinformatik weiter ausgebaut sowie auch insbesondere in den Monodisziplinen vermehrt Wert auf interdisziplinäre Ausbildungsaspekte gelegt werden. Denn der drohenden Betriebsblindheit kann insbesondere bei zukünftigen Spezialisten einer Monodisziplin nur wirksam begegnet werden, wenn diese in ihrer Ausbildung ein grundlegendes Verständnis für angrenzende Themengebiete anderer Disziplinen und für fachübergreifende Zusammenhänge erwerben.

Und was kann die Wirtschaftsinformatik im Speziellen dazu beitragen? Bezüglich der fachlichen Ausbildung ist die Wirtschaftsinformatik als Interdisziplin an der Schnittstelle zwischen BWL und Informatik einerseits sowie zwischen Forschung und Praxis andererseits hervorragend aufgestellt und braucht keinen Vergleich mit reinen Monodisziplinen zu scheuen. Allerdings wäre auch hier ein höherer Frauenanteil nicht nur in der Ausbildung an der Universität, sondern auch in Wissenschaft und Praxis wünschenswert. So bezifferte das statistische Bundesamt den bundesweiten Anteil weiblicher Studentinnen der Wirtschaftsinformatik im Wintersemester 2007/2008 auf knapp $14 \%$. Bei den Doktorand(inn)en ist der Anteil schon niedriger, bei den Habilitand(inn)en/Professor(inn)en noch geringer. Auch hier besteht noch dringender Handlungsbedarf, um den oben beschriebenen Herausforderungen gerecht zu werden. ${ }^{1}$

Hans Ulrich Buhl

\section{Literatur}

Haas S, Kur D (2009) Jeder dritte Vorstand eine Frau. Süddeutsche Zeitung (2009-10-28)

Jenkins P (2009) Investmentbanken basteln wieder. Financial Times Deutschland (2009-07-06) oV (2003) Homo sapiens - Die Wiege stand doch in Afrika. http://www.spiegel.de/wissenschaft/ mensch/0,1518,252492,00.html. Accessed 2003-06-12

oV (2009a) Gemischte Teams sind ertragsstärker. Technologie \& Management 58(5):30-31

oV (2009b) ILO: Wirtschaftskrise hat weltweit 20 Millionen Jobs vernichtet. http://de.reuters. com/article/economicsNews/idDEBEE5B605220091207. Accessed 2009-12-07

oV (2009C) Krise kostet laut Studie 10 Billionen Dollar. http://www.faz.net/s/Rub58241E4D F1B149538ABC24D0E82A6266/Doc E46B727E834A44EB5A2522BBC5D15BE1F ATpl Ecommon Scontent.html. Accessed 2009-08-29

oV (2009d) Risikofaktor Testosteron. http://www.ftd.de/karriere-management/karriere/: finanzgebaren-risikofaktor-testosteron/50003792.html. Accessed 2009-09-01

oV (2009e) Gottes Werk und Teufels Beitrag. http://www.sueddeutsche.de/finanzen/483/493826/ text/. Accessed 2009-11-09

Öchsner T (2009) Die Arbeitswelt wird älter, weiblicher, bunter, innovativer. Süddeutsche Zeitung $(2009-11-12 / 13)$

Rost K, Osterloh M (2008) You pay a fee for strong beliefs: homogeneity as a driver of corporate governance failure. http://ssrn.com/abstract=1304719. Accessed 2009-11-23

Schäfer U (2008) Die Wall Street hat den Krieg verloren. Süddeutsche Zeitung (2008-08-23/24)

Stauber-Klein B (2006) Frauen im Vorstand: Fehlanzeige. Westdeutsche Allgemeine Zeitung (2006-12-01)

Thorborg H (2009) Mehr Frauen weniger Ärger. Financial Times Deutschland (2009-06-22)

\footnotetext{
${ }^{1}$ An dieser Stelle möchte ich ganz herzlich Frau Dr. Julia Heidemann, M.Sc. with honors (FIM), und Herrn Dipl.-Inf. Peter Bartmann für ihre umfassende Unterstützung bei der Erstellung dieses Editorials und für ihre zahlreichen inhaltlichen Beiträge danken.
} 